\section{SEX- AND AGE-DIFFERENCES IN NATIVE T1 RELAXATION TIMES IN HEALTHY ADULTS AT 1.5 AND 3.0 TESLA}

${ }^{1} \mathrm{~S}$ Rauhalammi, ${ }^{1} \mathrm{P}$ Hall Barrientos, ${ }^{2} \mathrm{D}$ Carrick, ${ }^{1} \mathrm{C}$ McComb, ${ }^{1,2} \mathrm{~K}$ Mangion, ${ }^{1} \mathrm{G}$ Clerfond, 1J McClure, 'A Radjenovic, ${ }^{1,2} \mathrm{C}$ Berry. ${ }^{1} \mathrm{BHF}$ Glasgow Cardiovascular Research Centre, University of Glasgow, UK; ${ }^{2}$ Golden Jubilee National Hospital, UK

\subsection{6/heartjnl-2015-307845.28}

Background Limited information is available on sex differences in myocardial T1 relaxation times over age ranges. We used cardiac magnetic resonance (CMR) imaging at two field strengths to assess myocardial T1.

Methods Healthy adults underwent CMR at 1.5 Tesla (T) (Avanto) and $3.0 \mathrm{~T}$ (Verio). T1 maps were acquired in three short axis slices, using an optimised MOLLI investigational prototype sequence (Siemens Healthcare WIP 448). Global mean $\mathrm{T} 1$, in milliseconds (ms), was calculated from evaluable regionsof-interest using 16-segment model.

Results 84 volunteers (43 male) underwent scans $1.4 \pm 1.4$ days apart. Because of artefacts related to cardio-respiratory motion and susceptibility effects, $47(3.9 \%)$ segments were excluded at $1.5 \mathrm{~T}$ and $81(6.3 \%)$ segments at $3.0 \mathrm{~T}$, with a preponderance occurring at the distal slice.

Age-related decrease in T1 was observed in females, whereas male T1 remained reasonably constant (Figure 1 and Table 1). At $1.5 \mathrm{~T}$, amongst those $<40$ years T1 was higher for females $(961.3 \pm 19.3 \mathrm{~ms})$ than males $(932.0 \pm 22.8 \mathrm{~ms}, \mathrm{p}<0.001)$ whereas there was no difference in those $\geq 60$ years $(937.2 \pm$ 28.7 vs. $934.3 \pm 24.3 \mathrm{~ms}$, respectively, $\mathrm{p}=0.807$ ). Results were similar at $3.0 \mathrm{~T}$; female $\mathrm{T} 1 \mathrm{was}$ higher at $<40$ years $(1166.0 \pm$ 41.7 vs. $1139.6 \pm 26.1 \mathrm{~ms}, \mathrm{p}=0.044)$, but not at $\geq 60$ years $(1151.0 \pm 39.3$ vs. $1124.3 \pm 31.6 \mathrm{~ms}, \mathrm{p}=0.087)$.

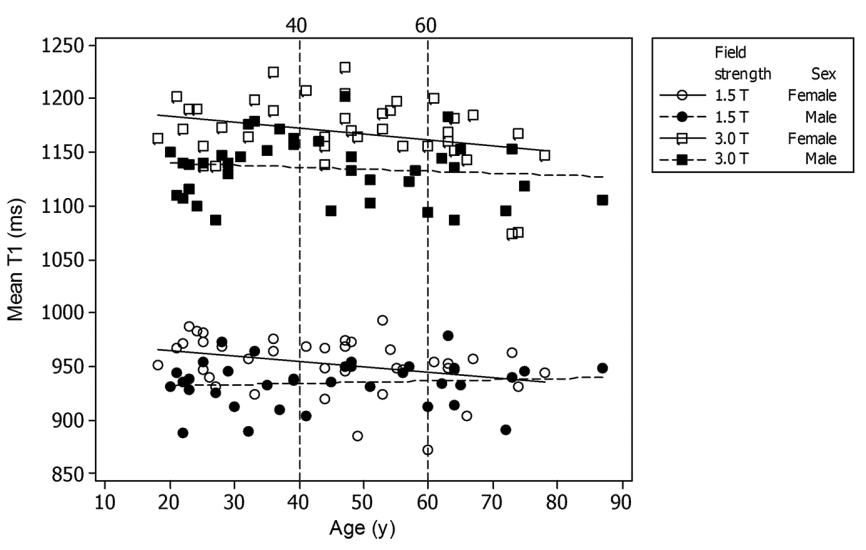

Abstract 28 Figure 1 Global myocardial T1 relaxation times.

Regression analysis shows that at $1.5 \mathrm{~T}$ average $\mathrm{T} 1$ decreases by $5.13 \mathrm{~ms}$ for each additional decade $(\mathrm{p}=0.038)$. An identical trend was observed at $3.0 \mathrm{~T}$, with regression coefficient $-0.564 \mathrm{~ms} /$ year approaching statistical significance $(\mathrm{p}=0.064)$.

Conclusions In healthy adults, sex difference in global myocardial mean T1 relaxation times are observed amongst younger. This pattern is consistent across CMR field strengths. Pre- vs. post-menopausal differences in myocardial structure and function of females may explain these differences and this possibility merits further assessment.
Abstract 28 Table 1 Global myocardial T1 relaxation times (mean $\pm \mathrm{SD}$, ms)

\begin{tabular}{lllll}
\hline & $1.5 \mathrm{~T}$ & \multicolumn{3}{l}{$3.0 \mathrm{~T}$} \\
\hline & Men & Women & Men & Women \\
\hline Age $<40, y(n=36)$ & $932.0 \pm 22.8$ & $961.3 \pm 19.3$ & $1139.6 \pm 26.1$ & $1166.0 \pm 41.7$ \\
Age 40-59, y $(n=25)$ & $939.8 \pm 16.5$ & $951.7 \pm 27.6$ & $1135.8 \pm 31.9$ & $1180.1 \pm 24.4$ \\
Age $\geq 60, y(n=23)$ & $934.3 \pm 24.3$ & $937.2 \pm 28.7$ & $1124.3 \pm 31.6$ & $1151.0 \pm 39.3$ \\
\hline
\end{tabular}

\section{SYNTHETIC ECV - SIMPLIFYING ECV QUANTIFICATION BY DERIVING HAEMATOCRIT FROM T1 BLOOD}

${ }^{1}$ TA Treibel * , 'M Fontana, ${ }^{2} \mathrm{~V}$ Maestrini, 'S Castelletti, 'S Rosmini, 'J Simpson, ${ }^{1} \mathrm{~A}$ Nasis ${ }^{1} \mathrm{H}$ Bulluck, ${ }^{1} \mathrm{~A}$ Abdel-Gadir, ${ }^{1} \mathrm{SK}$ White, ${ }^{1} \mathrm{C}$ Manisty, ${ }^{3} \mathrm{P}$ Kellman, ${ }^{4} \mathrm{~EB}$ Schelbert, ${ }^{5}$ MD Robson, ${ }^{5}$ SK Piechnik, ${ }^{1} \mathrm{JC}$ Moon. ${ }^{1}$ The Heart Hospital, University College London Hospitals, London, UK; ${ }^{2}$ Sapienza University, Rome, Italy; ${ }^{3}$ National Heart, Lung, and Blood Institute, National Institutes of Health, Bethesda, USA; ${ }^{4}$ UPMC Heart and Vascular Institute, University of Pittsburgh, Pittsburgh, USA; ${ }^{5}$ John Radcliffe Hospital, University of Oxford, Oxford, UK

\subsection{6/heartjnl-2015-307845.29}

Background Extracellular volume (ECV) quantification by cardiovascular magnetic resonance (CMR) measures the extracellular space. Current methodologies require blood haematocrit (Hct) correction, a barrier to easy clinical use. We hypothesised that the relationship between Hct and longitudinal relaxation time of blood $\left(\mathrm{T} 1_{\text {blood }}\right)$ could be calibrated and used to generate a synthetic ECV without Hct.

\begin{tabular}{|c|c|c|c|}
\hline & Derivation & Validation & $p$-value \\
\hline Total & 214 & 213 & \\
\hline Male & 107 & 104 & 0.8 \\
\hline Age & $60 \pm 15$ & $60 \pm 15$ & 0.6 \\
\hline $\mathrm{BSA}\left(\mathrm{m}^{2}\right)$ & $1.87 \pm 0.23$ & $1.87 \pm 0.23$ & 0.8 \\
\hline Healthy Volunteer & 33 & 33 & \\
\hline Aortic Stenosis & 62 & 61 & \\
\hline Cardiac Amyloidosis & 37 & 37 & \\
\hline Hypertrophic Cardiomyopathy & 34 & 34 & \\
\hline Anthracycline & 48 & 48 & \\
\hline \multicolumn{4}{|l|}{ Cardiac } \\
\hline $\operatorname{EDVi}\left(\mathrm{ml} / \mathrm{m}^{2}\right)$ & $71 \pm 21$ & $70 \pm 20$ & 0.9 \\
\hline $\operatorname{ESVi}\left(\mathrm{ml} / \mathrm{m}^{2}\right)$ & $25 \pm 14$ & $24 \pm 13$ & 0.5 \\
\hline LV mass index $\left(\mathrm{g} / \mathrm{m}^{2}\right)$ & $90 \pm 35$ & $92 \pm 35$ & 0.5 \\
\hline $\mathrm{SVi}\left(\mathrm{ml} / \mathrm{m}^{2}\right)$ & $47 \pm 12$ & $47 \pm 13$ & 0.7 \\
\hline $\operatorname{LVEF}(\%)$ & $66 \pm 12$ & $67 \pm 12$ & 0.7 \\
\hline LAAi $\left(\mathrm{cm}^{2} / \mathrm{m}^{2}\right)$ & $14 \pm 3$ & $14 \pm 5$ & 0.5 \\
\hline RAAi $\left(\mathrm{cm}^{2} / \mathrm{m}^{2}\right)$ & $12 \pm 4$ & $12 \pm 4$ & 0.9 \\
\hline \multicolumn{4}{|l|}{ Clinical } \\
\hline Hematocrit & $0.40 \pm 0.04$ & $0.40 \pm 0.04$ & 0.4 \\
\hline Createnine & $79 \pm 24$ & $78 \pm 21$ & 0.9 \\
\hline eGFR & $80 \pm 23$ & $78 \pm 22$ & 0.4 \\
\hline Systolic BP (mmHg) & $110 \pm 44$ & $108 \pm 49$ & 0.7 \\
\hline Diastolic BP (mmHg) & $65 \pm 28$ & $62 \pm 31$ & 0.5 \\
\hline \multicolumn{4}{|l|}{ T1 Mapping } \\
\hline SHMOLLI ECV (\%) & $33 \pm 10$ & $33 \pm 11$ & 0.9 \\
\hline
\end{tabular}

$B S A$, body surface area; $B P$, blood pressure; ECV, extracellular volume fraction; EDVi, indexed end-diastolic volume; ESVi, indexed end-systolic volume; eGFR, estimated glomerular filtration rate; LAAi, indexed left atrial area; LVEF, left ventricular ejection fraction; RAAi, indexed right atrial area; ShMOLLI, Shortened Modified Look-Locker Inversion recovery; SVi, indexed stroke volume 
Methods 427 subjects with a wide range of health and disease were divided into derivation $(\mathrm{n}=214)$ and validation $(\mathrm{n}=213)$ cohorts (Table 1 for patient characteristics). All subjects underwent T1 mapping with ShMOLLI at 1.5 Tesla for ECV quantification. Venous blood for Hct was obtained prior to scanning with 44 patients having a repeat Hct within $6 \mathrm{~h}$.

$\mathrm{ECV}$ was calculated as: $\mathrm{ECV}=\left(\Delta\left[1 / \mathrm{T} 1_{\text {myo }}\right] / \Delta\left[1 / \mathrm{T} 1_{\text {blood }}\right]\right) *$ [1-haematocrit]).

Synthetic Hct was approximated from the linear relationship between Hct and native $\mathrm{T} 1_{\text {blood }}$, and used to calculate synthetic ECV. Histological validation was performed on 18 patients with severe aortic stenosis (age $71 \pm 10$ years, $78 \%$ male). ECV was compared with collagen volume fraction from intra-operative biopsies taken during surgical valve replacement.

Results In the derivation cohort, native $\mathrm{T} 1_{\text {blood }}$ and Hct showed a linear relationship $\left(\mathrm{R}^{2}=0.45 ; p<0.001\right.$, Figure 1$)$. This was used to derive synthetic Hct $=0.88-\left(\mathrm{T} 1_{\text {blood }} /\right.$ 3240). Synthetic ECVcorrelated well with ECV $\left(\mathrm{R}^{2}=0.99\right.$; $p<0.001)$. These results were maintained in the validation cohort. Test:retest variability of haematocrit was higher than expected ( $n=44$, variability $10 \%$ with Hct:Hct $R^{2}=0.86$ ). On histological validation, synthetic and conventional ECV both correlated well with collagen volume fraction $\left(\mathrm{R}^{2}=0.61\right.$ and $0.69, p<0.001)$.

Conclusion Synthetic ECV allows instantaneous non-invasive quantification of the myocardial extracellular space without blood sampling. Inline application of synthetic ECV may be an attractive alternative in clinical practice.

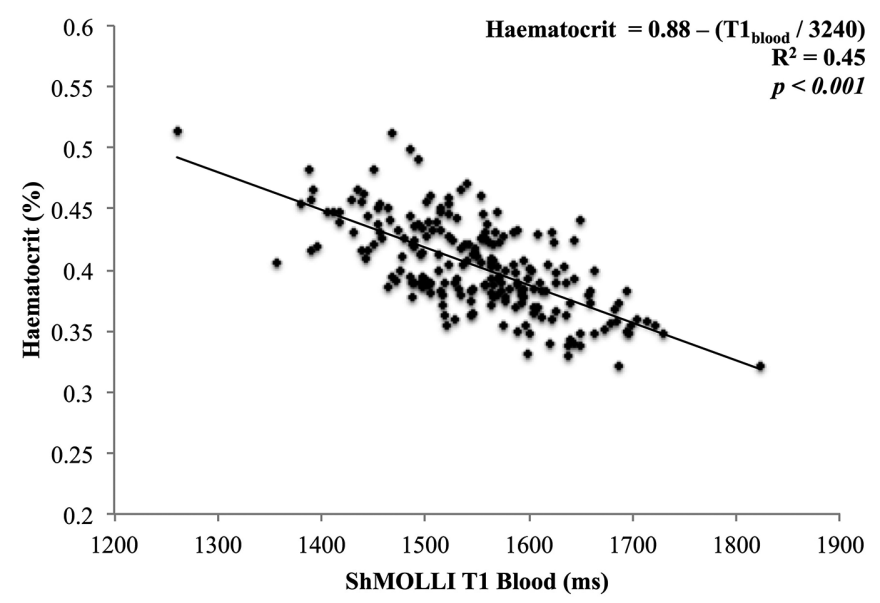

Abstract 29 Figure 1 Correlation between T1 blood and haematocrit. In the derivation cohort $(\mathrm{n}=214)$, native $\mathrm{T1}$ blood and hematocrit $(\mathrm{Hct})$ showed a linear relationship ( $R 2=0.45 ; p<0.001)$. This was used to derive synthetic $\mathrm{Hct}=0.88-\left(\mathrm{T} 1_{\text {blood }} / 3240\right)$.

\section{WHOLE BODY CONTRAST ENHANCED MRA CAN QUANTIFY AND MONITOR ATHEROSCLEROSIS PROGRESSION}

${ }^{1} \mathrm{JR}$ Weir-McCall, ${ }^{2} \mathrm{RD}$ White, ${ }^{3} \mathrm{SJ}$ Gandy, ${ }^{4} \mathrm{PG}$ Ramkumar, ${ }^{1} \mathrm{JJF}$ Belch, ${ }^{1} \mathrm{AD}$ Struthers, $1,4 \mathrm{JG}$ Houston. ${ }^{1}$ Division of Cardiovascular and Diabetes Medicine, Medical Research Institute, University of Dundee, DD1 9SY, UK; ${ }^{2}$ Department of Clinical Radiology, University Hospital of Wales, Cardiff, CF14 4XW, UK; ${ }^{3}$ Medical Physics, Ninewells Hospital, Dundee, DD1 9SY, UK; ${ }^{4}$ Clinical Radiology, Ninewells Hospital, Dundee, DD1 9SY, UK

10.1136/heartjnl-2015-307845.30
Aim To determine the ability of whole body magnetic resonance angiography (WB-MRA) to measure global atheroma burden progression.

Methods 50 consecutive patients with symptomatic peripheral arterial disease referred for clinical MRA were recruited. WB-MRA was performed at baseline, 6 months and 3 years. WB-MRA data was analysed by dividing the vasculature into 31 anatomical arterial segments. Each segment was scored according to degree of luminal narrowing: $0=$ normal, $1=<50 \%, 2=50-70 \%, 3=71-99 \%, 4=$ vessel occlusion. From this a standardised atheroma score (SAS) was calculated with a maximum score of 100 and minimum score of 0 . Progression was assessed with repeat measure ANOVA.

Results 36 patients were scanned at 0 and 6 months, with 26 patients scanned at the three year follow up. Only those who completed all 3 visits were included in the final analysis. At 3 years, $\mathrm{n}=18$ demonstrated atheroma progression while $\mathrm{n}=8$ showed stable or improved disease. Those with no progression had significantly lower baseline SAS, and were more likely to be on statin therapy ( $p<0.05$ for both). Baseline SAS was $15.7 \pm 10.3$ at baseline with no progression at 6 months $(\mathrm{SAS}=16.4 \pm 10.5, \mathrm{p}=0.67)$. At 3 years there was significant progression in atheroma $(\mathrm{SAS}=17.7 \pm 11.5, \mathrm{p}=0.01)$ (Figure 1). On multiple linear regression, age ( $\beta \quad 0.14$ $\mathrm{p}=0.014)$, pulse pressure $(\beta-0.12 \mathrm{p}=0.005)$ and anklebrachial pressure index $(\beta-7.7 \mathrm{p}=0.036)$ were independently associated with the rate of progression.

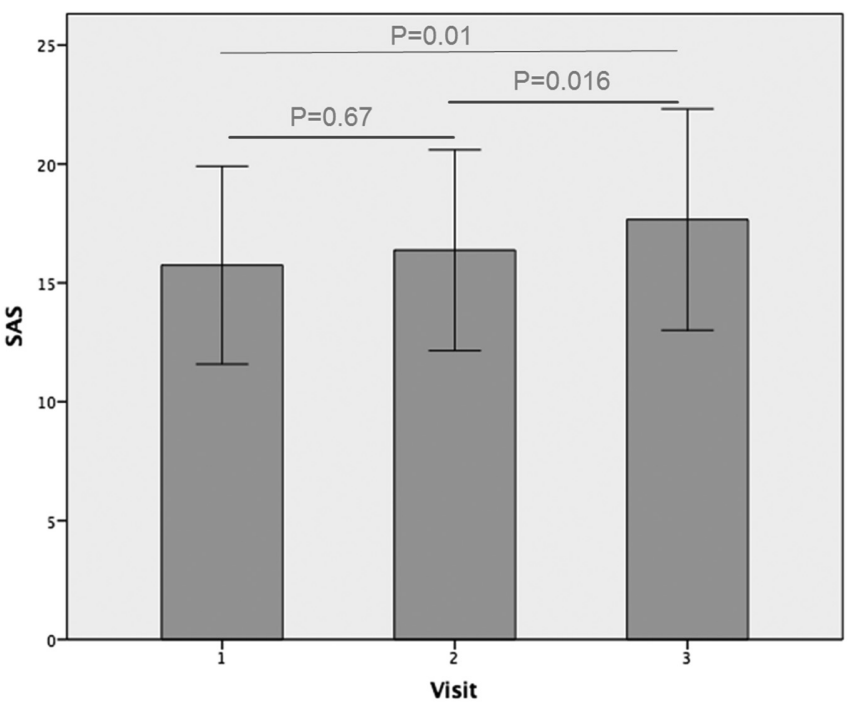

Abstract 30 Figure 1 Comparison of atheroma score at baseline, 6 months and 3 years. Visit $1=$ Baseline, Visit $2=6$ months, Visit $3=$ 3 years. T-bars represent $95 \%$ confidence intervals.

Conclusion Whole body contrast enhanced MRA can quantify and monitor atherosclerosis progression at 3 year follow-up even in a small cohort.

\section{ISCHAEMIA AND VIABILITY ASSESSMENT WITH ADENOSINE STRESS CMR IN HIGH RISK PATIENTS: SAFETY, FEASIBILITY AND TOLERABILITY}

Catherine Wilson, Alexander Carpenter, Amardeep Ghosh Dastidar, Jonathan Rodrigues, Nauman Ahmed, Anna Baritussio, Chris Benny Lawton, Elisa McAlindon, Chiara BucciarelliDucci. NIHR Bristol Cardiovascular Biomedical Research Unit, Bristol Heart Institute, Bristol, UK 